\title{
ST-elevation during head up tilt test: a challenging case in syncope unit
}

\author{
Andreina Carbone, Vincenzo Avitabile, Paolo Golino, Gerardo Nigro, Vincenzo Russo \\ Syncope Unit, Department of Medical Translational Sciences, University of Campania "L. Vanvitelli", Monaldi \\ Hospital, Naples
}

\begin{abstract}
A 65-year-old woman, during an elective head up tilt test, after the sublingual nitrate administration, experienced electrocardiogram alteration with ST-elevation in the inferior leads, that returned normal when the patient was laid supine after few minutes. Serial cardiac markers were not elevated and coronary angiography revealed normal epicardial coronary arteries.
\end{abstract}

Correspondence: Vincenzo Russo, Department of Translational Medical Sciences, University of Campania "L. Vanvitelli", Monaldi Hospital, P.zzale E. Ruggeri, 80131 Naples, Italy.

E-mail: v.p.russo@libero.it

Contributions: All the authors made a substantive intellectual contribution, performed part of the experiments. All the authors have read and approved the final version of the manuscript and agreed to be accountable for all aspects of the work.

Conflict of interests: The authors declare that they have no competing interests, and all authors confirm accuracy.

Funding: This research did not receive any specific grant from funding agencies in the public, commercial, or not-for-profit sectors.

Availability of data and materials: All data underlying the findings are fully available.

Ethics approval and consent to participate: No ethical committee approval was required for this case report by the Department, because this article does not contain any studies with human participants or animals. Informed consent was obtained from the patient included in this study.

Consent for publication: The patient gave her written consent to use her personal data for the publication of this case report and any accompanying images.

Key words: Head up tilt test, ST-elevation, electrocardiogram, vasovagal syncope, nitrates.

Received for publication: 15 March 2020.

Accepted for publication: 10 April 2020.

${ }^{\circ}$ Copyright: the Author(s), 2020

Licensee PAGEPress, Italy

Monaldi Archives for Chest Disease 2020; 90:1271

doi: 10.4081/monaldi.2020.1271

This article is distributed under the terms of the Creative Commons Attribution Noncommercial License (by-nc 4.0) which permits any noncommercial use, distribution, and reproduction in any medium, provided the original author(s) and source are credited.
Paradoxical vasospastic response to nitrates in vasospastic angina patients represents a rare but very challenging condition and the best therapeutic approach in this subgroup of patients remains unclear.

\section{Introduction}

Head up tilt test (HUTT) is a useful and safe test for the diagnosis of vasovagal syncope (VVS) in patients with suggestive history of reflex neutrally-mediated syncope [1]. Electrocardiographic alterations can rarely occur during HUTT and might be associated to various conditions, pathological or not [2]. In this case report we described an unusual electrocardiographic response to HUTT and discuss the possible physiopathological mechanism.

\section{Case report}

A 65-year-old woman with arterial hypertension, dyslipidemia, previous cerebral ischemic stroke was referred to our Syncope Unit for recurrent syncopal episodes of uncertain etiology. She denied any history of chest pain, palpitation or shortness of breath. The medical treatment included Olmersartan 40 mg once daily (OD); Atorvastatin $80 \mathrm{mg}$ OD; Cardioaspirin 100 $\mathrm{mg}$. The twelve-leads electrocardiogram (ECG) was normal. The orthostatic hypotension test and carotid sinus massage were negative. The transthoracic echocardiography showed normal ejection fraction (EF) calculated by the Simpson's biplane method (about 55\%) and mild mitral regurgitation. A HUTT was performed according to the Italian protocol [3] with continuous ECG monitoring and blood pressure measurements every $3 \mathrm{~min}$. The baseline value of $\mathrm{BP}$ and $\mathrm{HR}$ were $115 / 75 \mathrm{mmHg}$ and 80 bpm, respectively. After $20 \mathrm{~min}$ drug-free passive orthostatic phase, 400 microgram of sublingual nitroglycerin spray was administered (BP: 125/70 mmHg, HR: 92 bpm). Three min later, the patient started complaining of dizziness, blurred vision, tingling and angina without experiences syncope. The blood pressure and heart rate were $90 / 55 \mathrm{mmHg}$ and $72 \mathrm{bpm}$, respectively. The ECG revealed $2 \mathrm{~mm}$ ST-segment elevation (STE) in inferior leads with reciprocal ST depression in the lateral leads (I, aVL) (Figure 1), which returned at isoelectric line when the patient was placed in supine position. High-sensitivity cardiac troponin I (hs-CTnI) and creatine kinase-MB mass concentration (CKMB mass) were measured baseline and remained stable $6 \mathrm{~h}$ after the event. For the high cardiovascular risk, the patient was admitted to the coronary care unit for evaluation of ischemia; coronary angiography revealed normal epicardial coronary arteries (Figure 2). Before discharge, a loop recorder (ILR) was 
implanted. At six months follow-up the patient denied further episodes of syncope and no significant electrocardiographic abnormalities or arrhythmias were detected by ILR.

\section{Discussion}

The HUTT is generally considered a safe exam utilized in the differential diagnosis of patients with unexplained syncope. Lifethreatening complications, such as myocardial infarction, ventricular tachycardia [4] are rare and usually a consequence of coronary artery spasm or marked decrease in arterial blood pres- sure [5]. The ST segment changes, in particular ST segment depression and T wave inversions, during HUTT were observed at a relatively high incidence (about $18 \%$ ), primarily in women, with no clinical significance [6]. The primary mechanism driving ST-segment changes in young and otherwise generally healthy patients might be the imbalance of autonomic tone [6] and, in some cases, the improper filter application in single lead ECG monitor [7].

Few case reports showed ST-segment alteration in patients underwent HUTT [2,8]. Colman et al. [2] reported a case of STelevation in inferior leads, with reciprocal ST-segment depression in the left lateral and anterior leads, in a 56-year-old woman after an uncomplicated HUTT; the nitroglycerine administration

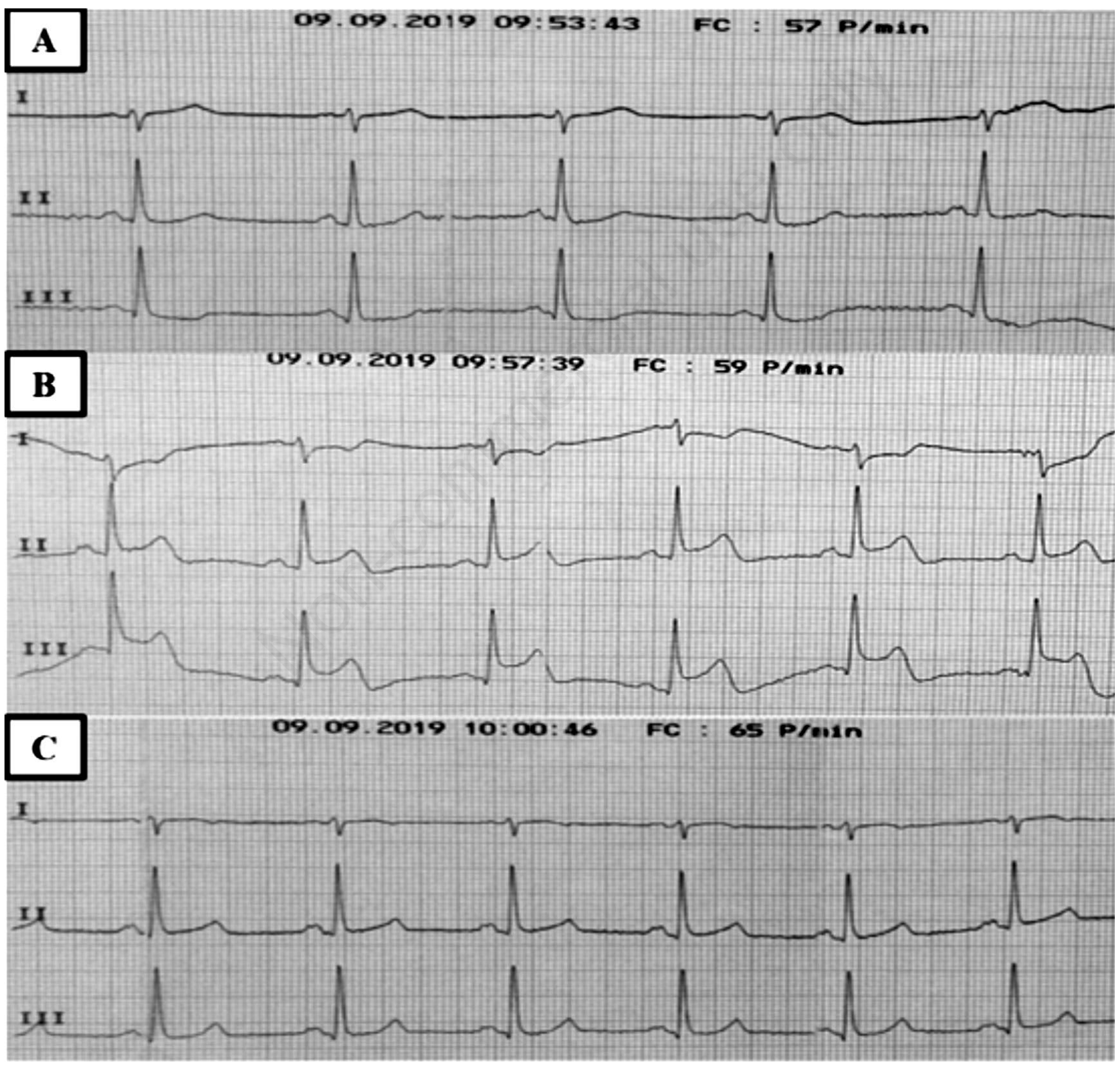

Figure 1. ECG before (A) and after (B) sublingual nitroglycerin administration. C) ECG after the resolution of symptoms. 

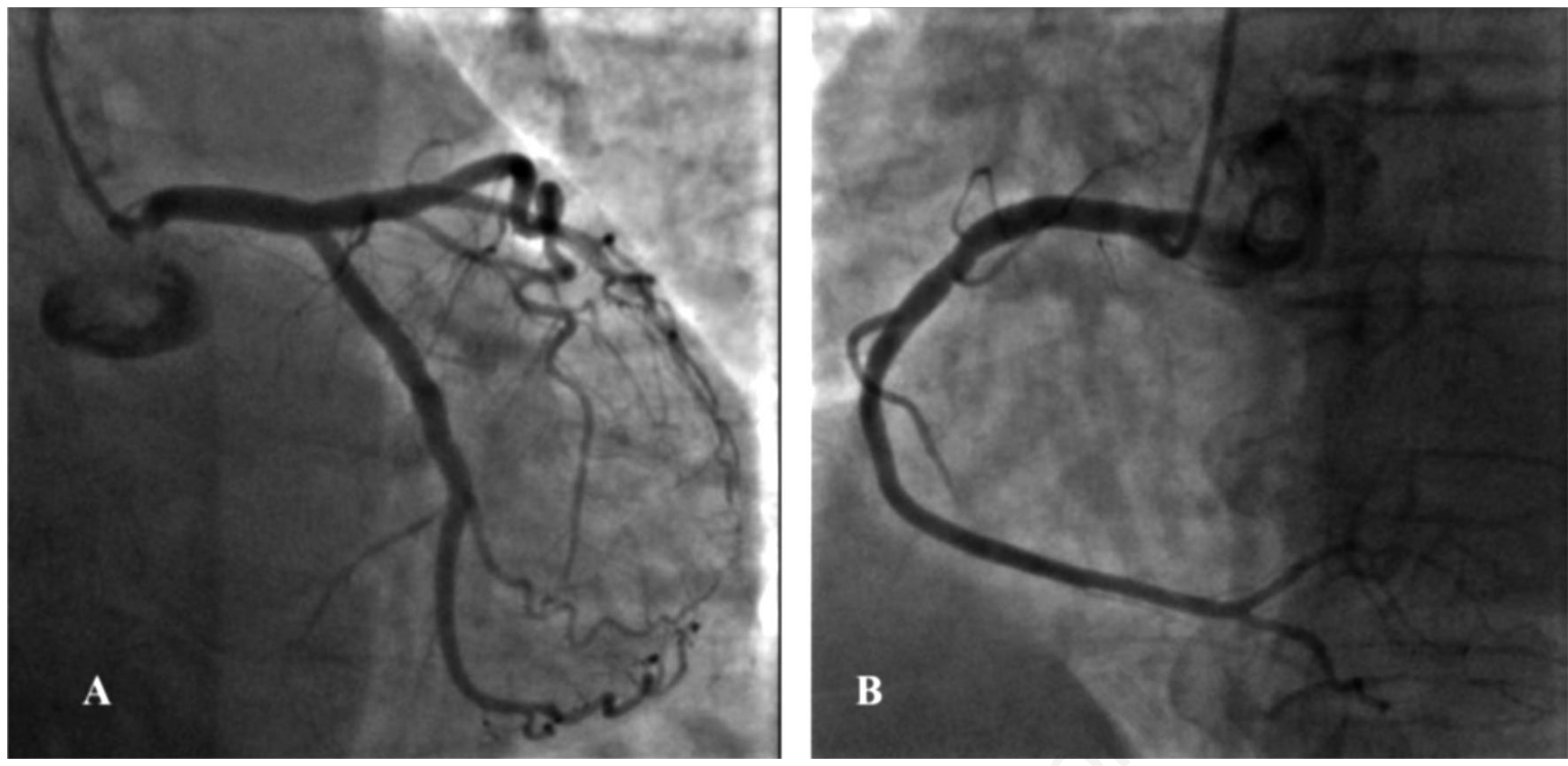

Figure 2. Coronary angiography with normal left (A) and right (B) coronary.

resolved instantly the ST-elevation. The coronary angiography showed no abnormalities. Omar et al. [8] described a case of ST elevation in the anterior leads during HUTT in a 57-year-old heavy-smoking man, without prior medical history, rapidly resolved after nitroglycerin therapy; serial cardiac markers were not elevated and coronary angiography revealed a nonocclusive $60 \%$ left anterior descending lesion. In both cases, the authors hypothesized that the underling mechanism was a coronary artery spasm [8].

The underling mechanisms of the HUTT-induced ST elevation is still debate, however the most likely cause seems to be the coronary vasoconstriction. HUTT-induced coronary spasm may be caused either by the acetylcholine direct stimulation of vascular smooth muscle of coronary arteries with endothelial damage or stenosis [9]; or by the augmented sympathetic activity which suddenly follows the HUTT induced parasympathetic tone [8]. Vasodilator drugs, as nitrates, usually reverse or prevent coronary artery spasm and its consequents [9].

Our case report is different from the previous reported in literature, because the ST elevation occurs after sublingual nitroglycerin administration during the hypotension and bradycardia related to the vasovagal response and it was accompanied by dizziness and angina.

Nitroglycerin can likewise markedly lower systemic vascular resistance, and may produce an appreciable reduction in coronary perfusion pressure. Such situation should be rare, since there would likely be a corresponding reduction in coronary vascular resistance, resulting in a facilitation of blood flow; however, nitroglycerin might have profound effects on diastolic blood pressure greater than the reduction in coronary vascular resistance, with the net effect of a decline in the transmyocardial coronary flow gradient and paradoxical ischemia [10]. Paradoxical vasospastic response to nitrates in vasospastic angina patients represents a rare but very challenging condition [5] and the best therapeutic approach in this subgroup of patients remains unclear $[5,10]$.

\section{Conclusions}

ST segment anomalies during HUTT might be related to a different condition, pathological or not. Paradoxical vasospastic response to nitrates during HUTT represents a rare but challenging condition. Rapid acting nitrates are usually effective for a rapid control of anginal episodes, but in rare case might be a trigger for trans-mural ischemia. The underlying mechanisms are not known and any firm conclusion about the best treatment strategy in this situation and the management is not possible.

\section{References}

1. Brignole M, Moya A, de Lange FJ, et al. 2018 ESC Guidelines for the diagnosis and management of syncope. Eur Heart J. 2018;39:1883-948.

2. Colman N, Wieling W, Wilde AA. A patient with recurrent syncope and ST-elevation on the electrocardiogram. Europace 2004;6:296-300.

3. Bartoletti A, Alboni P, Ammirati F, et al. [The tilt test enhanced with oral nitroglycerin in patients with unexplained syncope: the Italian Protocol. The Working Group on Syncope of the Associazione Italiana di Aritmologia e Cardiostimolazione-the Arrhythmia Area of the Associazione Nazionale Medici Cardiologi Ospedalieri].[Article in Italian]. Ital Heart J Suppl 2000;1:226-31.

4. Yiu KH, Tse HF, Lau CP. Ventricular tachycardia complicating head-up tilt test: the role of coronary artery spasm. Pacing Clin Electrophysiol 2011;34:e109-11.

5. Feldman RL, Pepine CJ, Conti CR. Unusual vasomotor coronary arterial responses after nitroglycerin. Am J Cardiol 1978;42:517-9. 
6. Vajapey R, Hutt Centeno E, Van Iterson EH, et al. ST-segment changes during tilt table testing for postural tachycardia syndrome: correlation with exercise stress test results. Clin Auton Res 2020;30:79-83.

7. Gupta A, Parakh N, Juneja R. Tilted ECG - An unusual tilt test response. Indian Pacing Electrophysiol J 2017;17:48-9.

8. Omar HR, Wang F, Fairbairn J. ST-segment elevation induced by coronary artery spasm during tilt-table test. Ther Adv Cardiovasc Dis 2013;7:274-5.

9. el-Tamimi H, Davies GJ, Crea F, et al. Response of human coronary arteries to acetylcholine after injury by coronary angioplasty. J Am Coll Cardiol 1993;21:1152-7.

10. Boden WE, Korr KS, Bough EW. Nifedipine-induced hypotension and myocardial ischemia in refractory angina pectoris. JAMA 1985;253:1131-5. 